\title{
MOTOR DE COMBUSTÃo INTERNA DE PISTÕES ROTATIVOS TRIANGULARES CURVILÍNEOS: WANKEL
}

\author{
Wandercleiton da Silva Cardoso ${ }^{1}$, Vitor Toniato Campana², Alex Borges do Nascimento ${ }^{3}$, \\ Fabiano Steiner Monteiro ${ }^{3}$, Franciely Costa Delunardo ${ }^{3}$, Gesiely Barcelos Matheus ${ }^{3}$ \\ 1. Mestre em engenharia de materiais e metalurgia pelo Instituto Federal do Espírito Santo e docente nível \\ superior dos cursos de engenharia da Faculdade Multivix. \\ 2. Graduando em engenharia civil pela Faculdade Multivix \\ 3. Graduando em engenharia mecânica pela Faculdade Multivix
}

\section{RESUMO}

O motor da combustão interna rotativo de pistões triangulares curvilíneos, que giram em cavidades troncoidais foi desenvolvido pelo engenheiro alemão Felix Wankel. Esse motor funciona segundo os quatro tempos tradicionais do ciclo Otto. A montadora de automóveis Mazda foi a empresa que mais utilizou o motor Wankel em seus carros esportivos RX-7 e $\mathrm{RX}$-8. No passado, várias empresam construíram automóveis com motores Wankel, tais como a NSU e a Citroen enquanto a Mercedes-Benz e GM fizeram protótipos veiculares com esta tecnologia. Algumas construtoras de motos como a Norton, a DKW Hercules e a Suzuki utilizam a tecnologia Wankel. Atualmente essa tecnologia é aplicada também em aviões e "hovercrafts", pelo seu baixo peso. Atualmente a utilização de motores Wankel é muito restrita na indústria automobilística, pois essas empresas estão receosas em investir nessa tecnologia em virtude de avanços tecnológicos do principal concorrente dos motores Wankel conhecido como motor quasiturbine que é a grande aposta do futuro.

Palavras-chave: Motor. Wankel. Combustão interna. Rotativo. Mazda.

\begin{abstract}
The internal combustion engine the and rotary combustion piston curvilinear triangular, revolving troncoidais cavities was developed by German engineer Felix Wankel. This engine works on the four traditional Otto cycle times. The automaker Mazda was the company that used the Wankel engine in his sports car RX-7 and RX-8. In the past several empresam built cars with Wankel engines, such as NSU and Citroen while Mercedes-Benz and GM made vehicle prototype with this technology. Some motorcycle builders like Norton, DKW Hercules and Suzuki used the Wankel technology. Currently this technology is also applied in aircraft and "hovercrafts" for its low weight. Currently the use of Wankel engines is very restricted in the automotive industry, as these companies are afraid to invest in this technology due to technological advances of the main competitor of Wankel engines known as Quasiturbine engine that is the big bet of the future.
\end{abstract}

Keywords: Engine. Wankel. Combustion. Internal. Rotary. Mazda.

\section{INTRODUÇÃO}

No ano de 1924 Felix Wankel desenvolveu o primeiro motor rotativo, e, desde então, dedicou-se para melhorar seu projeto. O objetivo era criar um motor que funcionasse de modo mais suave e silencioso, devido a sua estrutura mais simples e com menos peças, comparado aos motores de pistão. Em 1951, Felix Wankel fez os primeiros contatos com os 
engenheiros da NSU para estudar os problemas de vedação de espaços irregulares. Esses estudos resultaram na descoberta de que um motor triangular com lados convexos, girando em uma câmara poderia desenvolver um verdadeiro ciclo de quatro tempos. (GORDON et al, 1997)

A primeira aplicação desse princípio foi na forma de um compressor para o motor NSU de $50 \mathrm{cc}$, com dois tempos, que iria estabelecer novos recordes mundiais em Utah, em 1956. O compressor rotativo capacitou esse pequeno motor a desenvolver $260 \mathrm{HP}$ por litro, atingindo uma velocidade de quase $160 \mathrm{~km} / \mathrm{h}$. (AMROUCHE et al, 1996)

Em 1958, Wankel fez um acordo com a companhia norte-americana de aviação CurtissWright para que unissem seus esforços nas tentativas de fabricação de um grande motor baseado nesses princípios. Mais tarde começaram os testes com carros dotados de motores Wankel, diferentes uns dos outros. Dessa época até 1963, o motor foi gradualmente tomando forma definitiva e então adaptado a um pequeno NSU de dois lugares, apresentado no Salão de Automóvel em Frankfurt, no outono de 1963. A partir daí, foi concedida licença para a Toyo-Kogyo Co. (atual Mazda), Citroen, Mercedes-Benz, General Motors, e alguns construtores de motos como a Norton, a DKW Hercules e a Suzuki. Também foi utilizado em aviões e "hovercrafts", pelo seu baixo peso.

Talvez o melhor exemplo seja o magnífico NSU RO 80, com dois rotores, que começou a ser produzido em série em outubro de 1967, sendo que a versão com a direção do lado direito foi introduzida no mercado inglês em fins de 1968. Com o passar dos anos, e também devido aos problemas apresentados pelo motor, apenas a Mazda continuo a usá-lo. E em 1978, a marca lançou o modelo de maior sucesso com o motor Wankel, o RX-7, conforme Figura 1.(AMROUCHE et al, 1996)

Figura 2 - Mazda RX-7

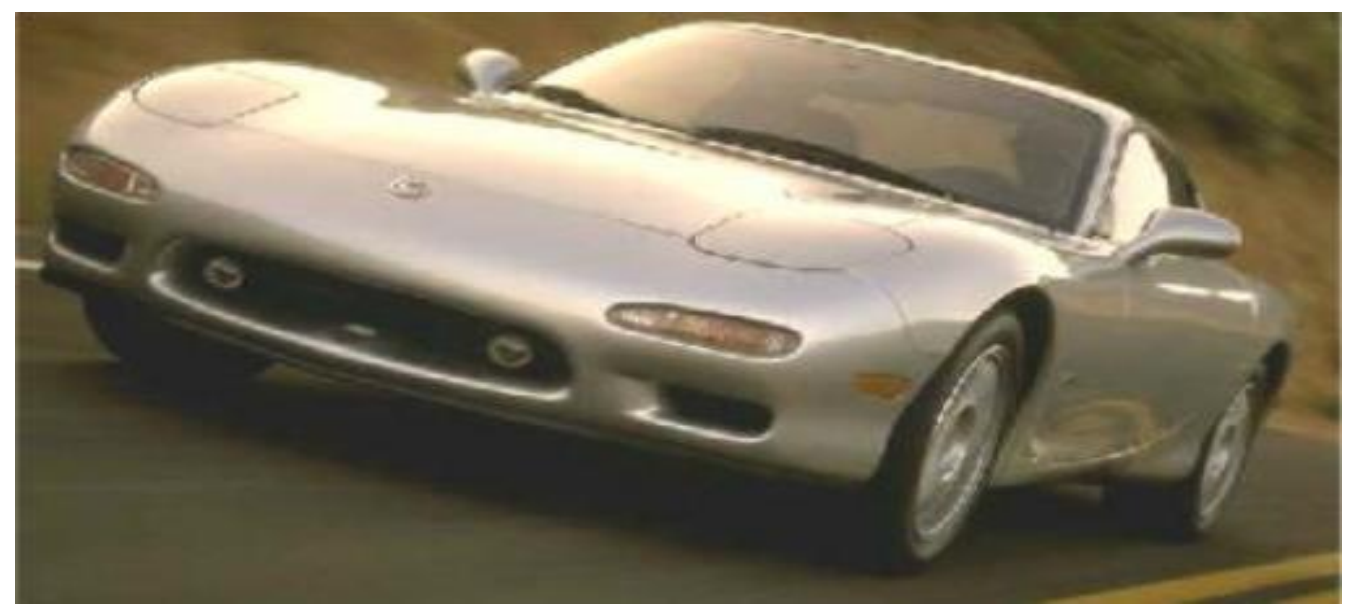

Devido ao consumo e emissões altas em relação aos concorrentes, a Mazda interrompeu a produção do RX-7 em 2002, mas a tecnologia ainda continuou sendo utilizada no RX-8, e recebeu o nome de Renesis, porém em 21 de junho de 2012, o último carro com o motor foi fabricado. Como a Mazda era a única que ainda utilizava o motor Wankel, este parou de ser fabricado permanentemente. (BADR, NAIK \& CALLAGHAN, 1991) 


\section{DESENVOLVIMENTO}

O bloco Wankel, consta apenas de: rotor, caixa de rotor e eixo de excêntricos. As partes do motor podem ser visualizadas abaixo, conforme figura 2 com detalhes, sendo que:

- Placa de Cobertura Primária

- Cobertura dos Rotores

- Placa de Cobertura Intermediária

- Placa de Cobertura Final

- Admissão Múltipla de Ar

- Admissão Múltipla de Mistura e Injeção (Ar e Gasolina)

- Admissão de Ar

- Engrenagens Estacionárias

- Rotores

- Cambota

- Saída do escape

Figura 2 - Partes do Motor Wankel

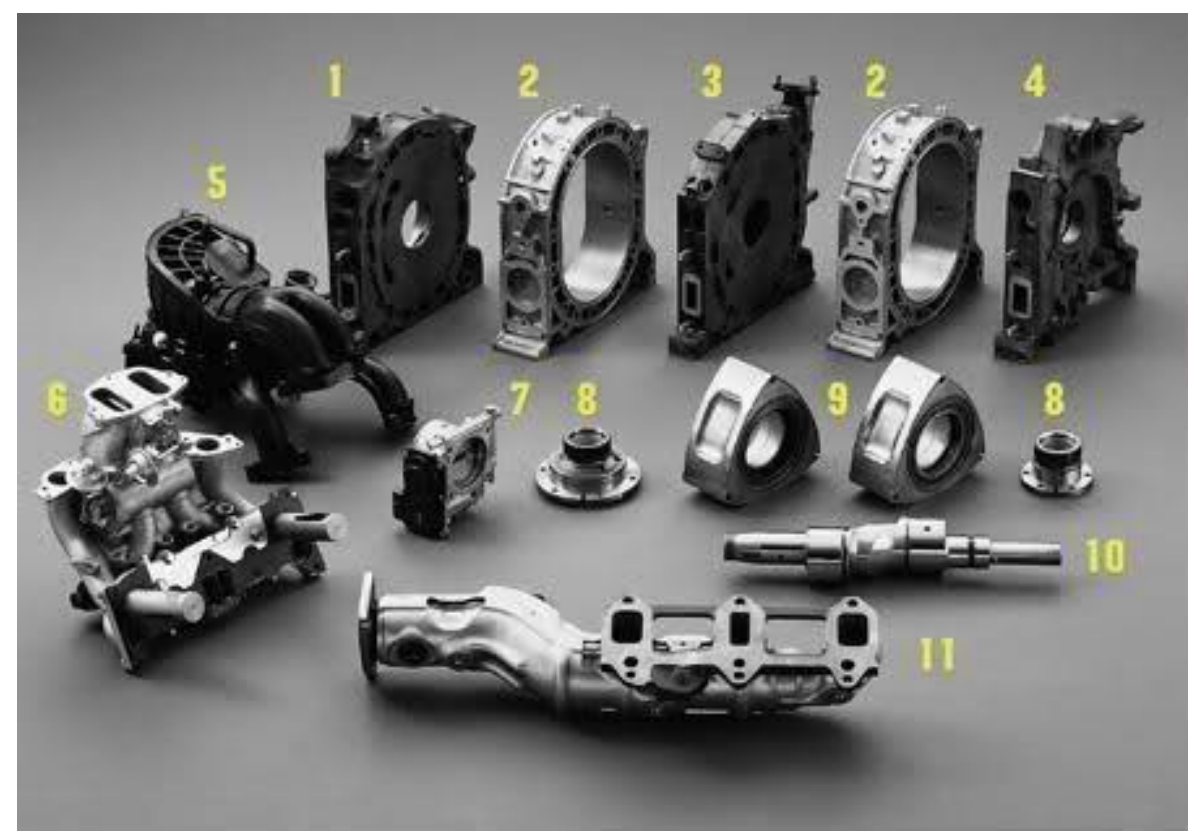

A carcaça externa ou bloco domotor Wankel conta com a superfície externa oval e alongada. Internamente as câmaras que abrigam o rotor que desempenha o papel do pistão também possuem forma oval. O rotor, por sua vez, é um componente com formato triangular 
e laterais abauladas. No centro deste triângulo localiza-se a engrenagem que se acopla à engrenagem do eixo de saída do motor. (BADR, NAIK \& CALLAGHAN, 1991)

A geometria interna do bloco faz com que o rotor ao girar (excentricamente) mantenha constantemente seus vértices em contato com as paredes do bloco, vedando cada uma das três câmaras formadas. Conforme esse giro ocorre, o volume de cada uma das três câmaras é alterado, produzindo aumento ou diminuição do volume dos gases do motor.

O funcionamento resume-se em quatro fases: admissão, compressão, combustão (ignição) e escapamento, conforme Figura 3.

Figura 3 - Quatro fases de funcionamento do motor Wankel

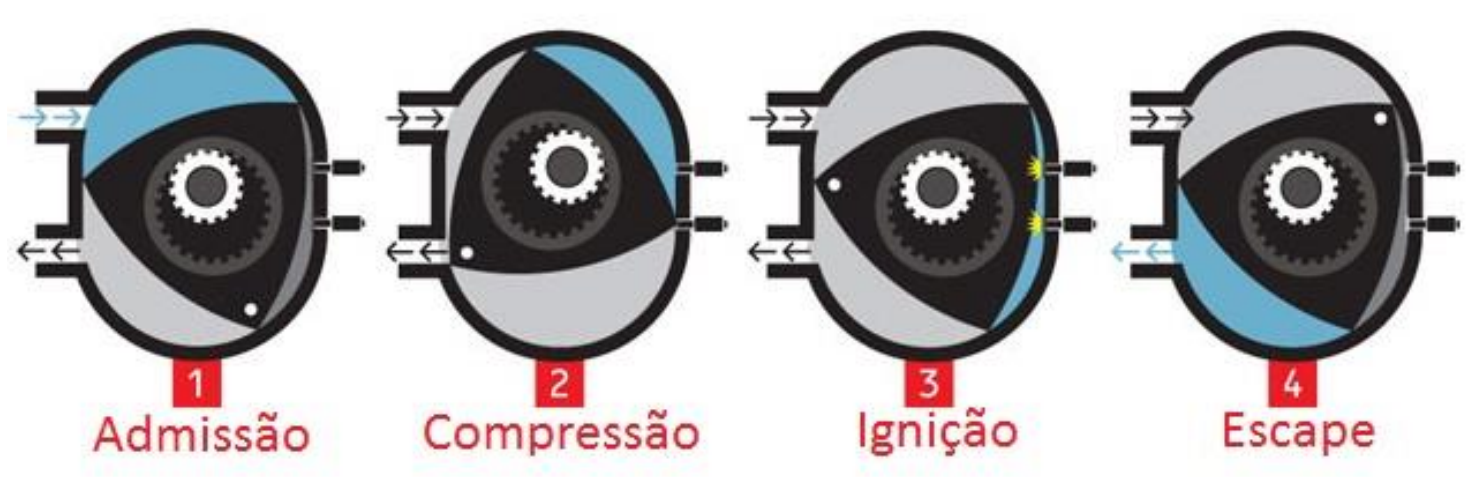

Na primeira fase, o rotor abre a janela de admissão. O seu movimento provoca a aspiração da mistura ar-combustível. Para que ocorra um maior enchimento da cavidade (melhor eficiência volumétrica), alguns motores Wankel são super carregados, ou seja, possuem compressor ou turbo para forçar a entrada da mistura durante a admissão. (JUNGBLUTH,1974)

Em seguida a janela de admissão é fechada pelo rotor e o volume começa a diminuir na fase de compressão. Essa fase dura até o espaço livre seja o mínimo (equivalente ao PMS Ponto Morto Superior - no motor alternativo).

Pouco depois, a mistura comprimida passa pela vela de ignição e uma centelha é gerada, inflamando a mistura. Em alguns motores são utilizadas duas velas para assegurar uma perfeita queima da mistura.

Com a combustão ocorre também a expansão dos gases, que ocasiona o deslocamento do rotor, e, consequentemente, a abertura da janela de escape, ocorrendo a liberação dos gases queimados. (GOLDBERG, 1971)

Em apenas uma volta do rotor, três ciclos completos ocorrem, bem como três voltas completas do eixo do motor, que equivale ao eixo do virabrequim no motor convencional. Esta relação de três voltas do eixo motor para cada volta do rotor se deve a relação das engrenagens. Nos motores convencionais, o virabrequim realiza duas voltas completas para cada ciclo completo do pistão. 
Fica então claro perceber, que devido às relações de voltas do rotor (em relação à engrenagem do motor) e número de explosões (uma em cada câmara, no total de três por volta) a cada giro completo do mesmo, porque os motores rotativos conseguem grandes níveis de potência com capacidades cúbicas razoavelmente pequenas.

Esse motor, de um modo geral, apresenta as seguintes vantagens relativamente aos motores alternativos:

- Simplicidade, devido à ausência de válvulas e mecanismos;

Eliminação dos mecanismos biela-manivela com redução dos problemas de compensação de forças e momentos, bem como vibratórios;

- Suavidade, que no caso do motor tradicional em que há inversão de movimento como no sobe e desce do pistão, no Wankel existe apenas o movimento rotativo, que proporciona um movimento mais suave;

- Menor número de peças móveis, o que poderá ocasionar construção e manutenção mais simples e de menor custo;

- Maior concentração de potência, logo menor volume e peso. Uma unidade de apenas 1,3 litro entrega $250 \mathrm{CV}$.

- Baixa vibração, devido ao fato de que só há um movimento rotativo, diminuindo, assim, o desgaste e prolongando sua vida útil.

Por outro lado, o motor apresenta problemas, como:

Problemas de vedação entre o cilindro e as paredes de cavidade, o que causa algumas dificuldades devido ao rigor das especificações do projeto e às tolerâncias mínimas de produção.

- Complexidade na lubrificação e refrigeração. Para complementar a lubrificação, uma pequena quantidade de óleo é queimada, o que eleva as emissões de poluentes, necessitando de um controle mais rigoroso na alimentação e no trato dos gases de escape.

- Curva de potência não muito elástica.

A câmara de combustão não é compacta (é extremamente longa), e acaba criando problemas de combustão (estabilidade de chama, que obriga ao uso de duas ou mesmo três velas) e a grandes perdas de calor, reduzindo o rendimento, e, consequentemente, aumentando o consumo.

O motor tem zonas intrinsecamente quente (entre as velas e a janela de escape) e outra intrinsecamente fria (zona após a admissão), apresentando grandes gradientes térmicos que têm de ser minimizados.

\section{CONCLUSÃO}


Devido aos grandes problemas enfrentados atualmente pelo aquecimento global, acreditamos que seja um dos principais motivos que o Motor Wankel não seja mais fabricado. Apesar de todas as comodidades para o usuário, como baixa vibração, o baixo som, as velocidades alcançadas, entre outras, as desvantagens superam essas vantagens.

A cada dia, novas tecnologias são produzidas pensando no conforto humano e no meio ambiente e baseado nos princípios do motor Wankel foi desenvolvido o motor quasiturbine, que veio para sanar suas desvantagens.

Todo o estudo de Felix Wankel foi grande contribuição para o desenvolvimento dessa e de novas tecnologias que ainda estão por vir.

\section{REFERÊNCIAS}

AMROUCH F. ,ERICKSON, P. , VARNHAGEN, S., "An experimental investigation of hydrogen-enriched gasoline in a Wankel rotary engine", International Journal of Hydrogen Energy, v.39,n.16,pp. 8525-8534, Feb.1996.

GORDON, R. PENNOCK, JOHN, BEAD ,E.," Force analysis of the apex seals in the wankel rotary compressor including the influence of fluctuations in the crankshaft speed", Mechanism and Machine Theory, v.32, n.3, pp. 349-361, Feb.1997.BADR , $\underline{0 .}$, NAIK , . , CALLAGHAN ,P.W., PROBERT, S.D. , "Rotary Wankel engines as expansion devices in steam Rankine-cycle engines",Applied Energy,v.39, n.1, pp. 59-76, Feb.1991.

BADR ,O., NAIK ,S. , CALLAGHAN ,P.W. , PROBERT, S.D. , "Wankel engines as steam expanders: Design considerations", v.40, n.3, pp. 157-170, Feb.1991.JUNGBLUTH G., "Biographie Dr.-Ing. E.h. Felix Wank", Mechanism and Machine Theory, v.9, n.2, pp. 145-146, Feb.1974.

GOLDBERG, M. "Rotary piston machines: Classification of design principles for engines, pumps and compressors" Journal of Mechanisms, v.6, n.3, pp. 342-343, Feb.1971. 